Pacific Journal of Mathematics

INVARIANT SUBSPACES OF COMPACT OPERATORS ON 


\title{
INVARIANT SUBSPACES OF COMPACT OPERATORS ON TOPOLOGICAL VECTOR SPACES
}

\author{
Arthur D. Grainger
}

\begin{abstract}
Let $(H, \tau)$ be a topological vector space and let $T$ be a compact linear operator mapping $H$ into $H$ (i.e., $T[V]$ is contained in a $\tau$-compact set for some $\tau$-neighborhood $V$ of the zero vector in $H$ ). Sufficient conditions are given for $(H, \tau)$ so that $T$ has a non-trivial, closed invariant linear subspace. In particular, it is shown that any complete, metrizable topological vector space with a Schauder basis satisfies the conditions stated in this paper. The proofs and conditions are stated within the framework of nonstandard analysis.
\end{abstract}

Introduction. This paper considers the following problem: given a compact operator $T$ (Definition 2.11) on a topological vector space $(H, \tau)$, does there exist a closed nontrivial linear subspace $F$ of $H$ such that $T[F] \subset F$ ? Aronszajn and Smith gave an affirmative answer to the above question when $H$ is a Banach space (see [1]). Also it is easily shown that the Aronszajn and Smith result can be extended to locally convex spaces. However, it appears that other methods must be used for nonlocally convex spaces.

Sufficient conditions are given for a topological vector space so that a compact linear operator defined on the space has at least one nontrivial closed invariant linear subspace (Definitions 2.1 and 4.1, Theorems 3.2, 4.2 and 4.7). In particular, it is shown that a Fréchet space with a Schauder basis satisfies the conditions given in this paper (Theorem 5.6). The basic techniques are an outgrowth of Bernstein's and Robinson's methods in [2], [3] and [4]; consequently, the proofs and arguments of the main results are stated within the framework of nonstandard analysis. It will be assumed that the reader is familiar with Abraham Robinson's book on Nonstandard Analysis [11] or W. A. J. Luxemburg's paper on Monad Theory [10].

1. Nonstandard topological vector spaces. Let us briefly examine the basic concepts of nonstandard topological vector space theory that are used in this paper. A more detailed discussion of nonstandard topological vector spaces can be found in [6] and [7]. Let $\mathrm{C}$ be the complex numbers, let $H$ be a vector space over $C$ and let $B_{\Gamma}$ be the full set theoretical structure of $H \cup C$. Throughout this paper $(H, \tau)$ will denote a complex topological vector space $H$ with topology 
$\tau$. Also we will assume that the nonstandard structure ${ }^{*} B_{\Gamma}$ of $H \cup C$ is a higher order ultrapower enlargement of $B_{\Gamma}$ (see [6], Chapter 1, §1).

The nonnegative integers, positive integers and the real numbers are designated by $\mathbf{N}, \mathbf{N}_{+}$and $\mathbf{R}$ respectively. Moreover, the map $x \mapsto^{*} x$ of $H \cup \mathbf{C}$ into ${ }^{*} H \cup{ }^{*} \mathbf{C}$ is the identity on $\mathbf{N}, \mathbf{R}, \mathbf{C}$ and $H$. As usual, the extensions to ${ }^{*} \mathbf{C}$ of the algebraic operations,$+ \cdot$ and $\mid$ on $\mathbf{C}$ are denoted by the same symbols. The same is true of the extension to ${ }^{*} \mathbf{R}$ of the ordering $<$ on $\mathbf{R}$.

An element $\lambda \in{ }^{*} \mathbf{C}$ is called an infinitesimal if and only if $|\lambda| \leqq \delta$ for each positive $\delta$ in $\mathbf{R}$ and finite if and only if $|\lambda| \leqq \delta$ for some positive $\delta \in \mathbf{R}$. If $\lambda \in{ }^{*} \mathbf{C}$ is not finite then $\lambda$ is called infinite. An element $\lambda \in *^{*} \mathbf{C}$ is called near-standard if there is an element in $\mathbf{C}$, denoted by ${ }^{\circ} \lambda$, such that $\lambda-{ }^{\circ} \lambda$ is an infinitesimal. It can be shown that ${ }^{\circ} \lambda$ exists and is unique if and only if $\lambda \in{ }^{*} \mathbf{C}$ is finite.

The extension to ${ }^{*} H$ of vector addition, + , on $H$ is again denoted by the same symbol and the scalar multiplication operation on ${ }^{*} \mathbf{C} \times{ }^{*} H$ takes $(\lambda, z)$ to $\lambda z$. For each $x$ in $H, \mu_{\tau}(x)$ is defined by

$$
\mu_{\tau}(x)=\cap\left\{* V \mid V \in \mathcal{N}_{\tau}(x)\right\}
$$

where $\mathcal{N}_{\tau}(x)$ is the filter of $\tau$-neighborhoods of $x$. Since $\mathcal{N}_{\tau}(x)=$ $\left\{V+x \mid V \in \mathcal{N}_{\tau}(0)\right\}$ it follows that

$$
\mu_{\tau}(x)=\mu_{\tau}(0)+x
$$

for every $x$ in $H$. It is easy to see that $\mu_{\tau}(0)+\mu_{\tau}(0)=\mu_{\tau}(0)$ and $\lambda \mu_{\tau}(0) \subset \mu_{\tau}(0)$ for finite $\lambda$ in ${ }^{*} \mathbf{C}$ ([6], Proposition I.6).

An element $z$ of ${ }^{*} H$ is called $\tau$-near standard if and only if $z \in \mu_{\tau}(x)$ for some $x$ in $H$ which is equivalent to $z-x \in \mu_{\tau}(0)$ for some $x$ in $H$. The set of all $\tau$-near-standard points of ${ }^{*} H$ will be denoted by $n s_{\tau}\left({ }^{*} H\right)$. It is easily shown that $n s_{\tau}\left({ }^{*} H\right)$ is closed under addition and multiplication by finite elements of ${ }^{*} \mathbf{C}$. If $\tau$ is Hausdorff then for each $z \in n s_{\tau}\left({ }^{*} H\right)$ there exists an unique point in $H$, denoted by ${ }^{\circ} z$, such that $z-{ }^{\circ} z \in \mu_{\tau}(0)$. Note that $H \subset n s_{\tau}\left({ }^{*} H\right)$.

For $A \subset^{*} H$, the $\tau$-standard part of $A, s t_{\tau}(A)$, is defined by

$$
s t_{\tau}(A)=\left\{x \in H \mid z \in \mu_{\tau}(x) \text { for some } z \in A\right\} .
$$

Consequently, $s t_{\tau}(A) \subset H$ for $A \subset{ }^{*} H$ and it can be shown that

$$
s t_{\tau}(* A)=\bar{A}
$$

for any $A \subset H$ ([6], Proposition I.2). Observe that $s_{\tau}(\{z\})=\left\{{ }^{\circ} z\right\}$ for each $z \in n s_{\tau}\left({ }^{*} H\right)$ if $\tau$ is Hausdorff. 
Another interesting concept, although not pertinent to the main objective, is the notion of a finite point. The set of $\tau$ finite points, $\operatorname{fin}_{\tau}(* H)$, is defined by

(1.5) $\operatorname{fin}_{\tau}\left({ }^{*} H\right)=\cap\left\{\alpha \mu_{\tau}(0) \mid \alpha \in{ }^{*} \mathbf{R}\right.$ is positive and infinite $\}$.

It can be shown that $z \in \mathrm{fin}_{\tau}\left({ }^{*} H\right)$ if and only if for $V \in \mathcal{N}_{\tau}(0)$ there exists an $n \in \mathbf{N}_{+}$such that $z \in *(n V)$ ([6], Corollary I.15). Furthermore, $n s_{\tau}\left({ }^{*} H\right) \subset \mathrm{fin}_{\tau}\left({ }^{*} H\right)$ and $\mathrm{fin}_{\tau}\left({ }^{*} H\right)$ is closed under addition and multiplication by finite elements of ${ }^{*} \mathbf{C}$ (see [6], Proposition I.16 or [7], Theorem 1.2).

Henson and Moore first explored the relationship between $\tau$ bounded subsets of $H$ and $\operatorname{fin}_{\tau}\left({ }^{*} H\right)$ by establishing the fact that $A \subset H$ is $\tau$-bounded if and only if $* A \subset$ fin $_{\tau}\left({ }^{*} H\right)$ ([7], Theorem 2.1). This idea can be generalized still further.

Proposition 1.6. If $A \subset{ }^{*} H$ is internal and $A \subset \mathrm{fin}_{\tau}\left({ }^{*} H\right)$ then $\mathrm{st}_{\tau}(A)$ is $\tau$-bounded.

Proof. Let $A \subset^{*} H$ be internal (i.e., $A \in * \mathscr{P}(H)$ ) and assume $A \subset \operatorname{fin}_{\tau}\left({ }^{*} H\right)$. Let $V \in \mathcal{N}_{\tau}(0)$ be closed and balanced and let $\phi\langle\mathscr{P}(H), \mathbf{R}, V\rangle$ designate the following sentence.

"If $X \in \mathscr{P}(H)$ such that $X \subset \delta V$ for some positive $\delta \in \mathbf{R}$ then either $X \subset \delta V$ for all positive $\delta \in \mathbf{R}$ or there exists a positive $\delta_{0} \in \mathbf{R}$ such that $X \subset \delta V$ for $\delta_{0}<\delta$ and $X \not \subset \delta V$ for $0<\delta<$ $\delta_{0} . "$

Since $V$ is balanced it follows that $\phi\langle\mathscr{P}(H), \mathbf{R}, V\rangle$ is true in $B_{\Gamma}$ and consequently $\phi\left\langle{ }^{*} \mathscr{P}(H),{ }^{*} \mathbf{R},{ }^{*} V\right\rangle$ is true in ${ }^{*} B_{\Gamma}$. Since $A \subset \mathrm{fin}_{\tau}\left({ }^{*} H\right)$, we have $A \subset \lambda^{*} V$ for positive, infinite $\lambda \in{ }^{*} \mathbf{R}$. If $A \subset \lambda^{*} V$ for each positive $\lambda \in{ }^{*} \mathbf{R}$ then $A \subset^{*} V$. If there exists a positive $\lambda_{0} \in{ }^{*} \mathbf{R}$ such that $A \subset \lambda^{*} V$ for $\lambda_{0}<\lambda$ and $A \not \subset \lambda^{*} V$ for $0<\lambda<\lambda_{0}$ then $\lambda_{0}$ is finite. Indeed, if $\lambda_{0}$ were infinite then $2^{-1} \lambda_{0}$ is infinite and $2^{-1} \lambda_{0}<\lambda_{0}$ which would imply $A \not \subset 2^{-1} \lambda_{0}{ }^{*} V$. But $A \subset$ fin $_{\tau}\left({ }^{*} H\right) \subset 2^{-1} \lambda_{0}{ }^{*} V$. In either case there exists a positive $\delta \in \mathbf{R}$ such that $A \subseteq$ $*(\delta V)$. Therefore, $\mathrm{st}_{\tau}(A)$ is $\tau$-bounded since $\mathrm{st}_{\tau}(A) \subset \mathrm{st}_{\tau}\left({ }^{*}(\delta V)\right)=\delta V$ by (1.4).

Finally, we need to consider the relationship between internal linear subspaces of ${ }^{*} H$ and linear subspaces of $H$. Let $\mathscr{F} \mathscr{S}(H)$ denote the collection of all finite dimensional linear subspaces of $H$. We will refer to elements of $* \mathscr{F} \mathscr{S}(H)$ as *-finite dimensional subspaces of ${ }^{*} H$. Also, let us symbolize ${ }^{\circ} F=\mathrm{st}_{\tau}(F)$ for $F \in * \mathscr{F} \mathscr{S}(H)$. 
PROPOSITION 1.7. If $(H, \tau)$ is a metrizable topological vector space and if $F \in * \mathscr{F} \mathscr{Y}(H)$ then ${ }^{\circ} F$ is a $\tau$-closed linear subspace of $H$.

By definition of $\mathscr{F} \mathscr{Y}(H)$, there exists $d: \mathscr{F} \mathscr{Y}(H) \rightarrow \mathbf{N}$ such that $d(F) \in \mathbf{N}$ is the dimension of $F$ for $F \in \mathscr{F} \mathscr{S}(H)$. Therefore ${ }^{*} d: * \mathscr{F} \mathscr{S}(H) \rightarrow{ }^{*} \mathbf{N}$ and ${ }^{*} d(F)$ is called the ${ }^{*}$-finite dimension of $F$ for $F \in * \mathscr{F} \mathscr{S}(H)$.

Proposition 1.8. Let $(H, \tau)$ be a Hausdorff topological vector space and let $F, F_{1} \in * \mathscr{F} \mathscr{S}(H)$ such that $F \subset F_{1}$. If $* d\left(F_{1}\right)=* d(F)+1$ then ${ }^{\circ} F C^{\circ} F_{1}$ and any two points of ${ }^{\circ} F_{1}$ are linearly dependent modulo ${ }^{\circ} \mathrm{F}$. I, §8.

The proofs of the above propositions may be found in [6], Chapter

2. Property 1. We now have the machinery needed to give sufficient conditions for a compact operator on the complex topological vector space $(H, \tau)$ to have a nontrivial $\tau$-closed invariant linear subspace.

Let $\mathscr{L}(H)$ denote the vector space of all linear transformations of $H$ into $H$. We will refer to elements of $* \mathscr{L}(H)$ as internal linear transformation of ${ }^{*} H$ into ${ }^{*} H$.

DEFINITION 2.1. A topological vector space $(H, \tau)$ is said to satisfy Property 1 if and only if there exist $P \in * \mathscr{L}(H)$ and $H_{\eta} \in^{*} \mathscr{F} \mathscr{S}(H)$ such that the following conditions hold.

(1) The internal linear transformation $P$ maps ${ }^{*} H$ into $H_{n}$.

(2) For $V \in \mathcal{N}_{\tau}(0)$ there exists $W \in \mathcal{N}_{\tau}(0)$ such that $P\left[{ }^{*} W\right] \subset^{*} V$.

(3) If $x \in H$ then $P(x)-x \in \mu_{\tau}(0)$.

We shall show that compact operators (Definition 2.11) on complex spaces which satisfy property 1 have nontrivial closed invariant linear subspaces.

Before discussing the proof of the above statement, let us first examine the influence of property 1 on a $\tau$-continuous linear transformation $T$, i.e., a linear operator, defined on $H$. The idea is to define $T^{\prime}=P^{*} T P$, where $P$ satisfies the condition of Property 1 , and show that if $F \subset H_{\eta}$ is a ${ }^{*}$-finite dimensional subspace such that $T^{\prime}[F] \subset F$ then $T\left[{ }^{\circ} F\right] C^{\circ} F$. Thus we will have a means of generating invariant subspaces for $T$.

Assume $(H, \tau)$ satisfies Property 1 and let $P \in * \mathscr{L}(H)$ and $H_{\eta} \in$ $* \mathscr{F} \mathscr{S}(H)$ be determined by Property 1 . Also throughout this section, it will be assumed that $(H, \tau)$ is Hausdorff. We see immediately 


$$
H=\mathrm{st}_{\tau}\left(H_{\eta}\right)={ }^{\circ} H_{\eta} \quad \text { and } \quad x={ }^{\circ}[P(x)]
$$

for $x \in H$. Also, Condition 2 of Definition 2.1 implies

$$
P\left[\mu_{\tau}(0)\right] \subset \mu_{\tau}(0) \text {. }
$$

PROPOSITION 2.4. If $z \in \mathrm{ns}_{\tau}\left({ }^{*} H\right)$ then $P(z) \in \mathrm{ns}_{\tau}\left({ }^{*} H\right),{ }^{\circ}[P(z)]={ }^{\circ} z$ and $z-P(z) \in \mu_{\tau}(0)$.

(See [6], Proposition II.2.)

Let $T$ be a linear operator on $H$, i.e., $T \in \mathscr{L}(H)$ and $T$ is continuous, and consider ${ }^{*} T$. It can be shown that

$$
* T\left[\mu_{\tau}(0)\right] \subset \mu_{\tau}(0)
$$

which implies

$$
{ }^{\circ}[* T(z)]=T\left({ }^{\circ} z\right)
$$

for $z \in \mathrm{ns}_{\tau}\left({ }^{*} H\right)$ (see [11], Theorem 4.2.7 or [6], Propositions I.4 and II.3). By defining

$$
T^{\prime}=P^{*} T P
$$

We obtain an internal linear transformation that maps ${ }^{*} H$ into $H_{\eta}$. Furthermore

$$
T^{\prime}\left[\mu_{\tau}(0)\right] \subset \mu_{\tau}(0)
$$

by (2.3) and (2.5). If a momentary abuse of language is permitted then we can say that the next proposition shows how well $T^{\prime}$ approximates $T$ on near-standard points.

Proposition 2.9. Let $T \in \mathscr{L}(H)$ be $\tau$-continuous. If $z \in \mathrm{ns}_{\tau}\left({ }^{*} H\right)$ then $T^{\prime}(z) \in \mathrm{ns}_{r}\left({ }^{*} H\right)$ and ${ }^{\circ}\left[T^{\prime}(z)\right]=T\left({ }^{\circ} z\right)$.

(See [6], Proposition II.5.)

As promised, the following proposition gives a useful way of generating invariant linear subspaces for $\tau$-continuous $T \in \mathscr{L}(H)$.

Proposition 2.10. Let $F \in * \mathscr{F} \mathscr{S}(H)$ such that $F \subset H_{\eta}$ and let $T \in \mathscr{L}(H)$ be $\tau$-continuous. If $T^{\prime}[F] \subset F$ then $T\left[{ }^{\circ} F\right] \subset{ }^{\circ} F$. 
Proof. If $x \in{ }^{\circ} F$ then $z-x \in \mu_{i}(0)$ for some $z \in F$ which implies ${ }^{\circ} z=x$. We infer $T(x)=T\left({ }^{\circ} z\right)={ }^{\circ}\left[T^{\prime}(z)\right]$ by Proposition 2.9. Therefore, $\quad T^{\prime}[F] \subset F$ implies $T^{\prime}(z) \in F$ which implies $T(x) \in$ ${ }^{\circ} F$. Consequently $T\left[{ }^{\circ} F\right] \subset{ }^{\circ} F$.

We are particularly concerned with the relationship between $T$ and $T^{\prime}$ when $T$ is compact. So let us now formally define compact operators.

Definition 2.11. Let $(H, \tau)$ be a topological vector space. A map $T \in \mathscr{L}(H)$ is called a compact operator if and only if there exists a $V \in \mathcal{N}_{\tau}(0)$ such that $T[V]$ is contained in a $\tau$-compact set.

Compact operators are continuous, a fact easily derived from the boundedness of compact sets. Equivalently $T \in \mathscr{L}(H)$ is compact if and only if the $\tau$-closure of $T[V]$ is compact for some $V \in \mathcal{N}_{\tau}(0)$ since $(H, \tau)$ is regular. Moreover, using Robinson's characterization of compact sets, we arrive at still another equivalent form of Definition 2.11 , i.e., $T \in \mathscr{L}(H)$ is compact if and only if ${ }^{*} T[* V] \subset \mathrm{ns}_{\tau}\left({ }^{*} H\right)$ for some $V \in \mathcal{N}_{\tau}(0)$ (see [11], Theorem 4.1.13). The next proposition shows that this idea is transferable to $T^{\prime}$.

Proposition 2.12. If $T \in \mathscr{L}(H)$ is compact then there exists a $W \in \mathcal{N}_{\tau}(0)$ such that $T^{\prime}\left[{ }^{*} W\right] \subset \mathrm{ns}_{\tau}\left({ }^{*} H\right)$.

Proof. If $\overline{T[V]}$ is compact for some $V \in \mathcal{N}_{\tau}(0)$ then

$$
{ }^{*} T\left[{ }^{*} V\right] \subset *(\overline{T[V]}) \subset \mathrm{ns}_{\tau}\left({ }^{*} H\right)
$$

(see [11], Theorem 4.1.13 or [6], Proposition I.1). By Condition 2 of Definition 2.1 , there exists $W \in \mathcal{N}_{\tau}(0)$ such that $P\left[{ }^{*} W\right] \subset^{*} V$ which implies ${ }^{*} T P\left[{ }^{*} W\right] \subset \mathrm{ns}_{\tau}\left({ }^{*} H\right)$. Therefore,

$$
T^{\prime}\left[{ }^{*} W\right]=P[* T P[* W]] \subset \mathrm{ns}_{\tau}\left({ }^{*} H\right)
$$

by Proposition 2.4 .

3. Invariant subspaces. As stated earlier, we want to use Property 1 (Definition 2.1) to produce a nontrivial, closed invariant linear subspace for compact operator $T$ on $H$. In the previous section, we established some interesting relationships between operators and Property 1; however, to give some motivation for defining Property 1 , let's examine the following outline of a nonstandard proof of the 
existence of a nontrivial, closed invariant linear subspace for a compact operator $T$ when we assume the underlying space $(H, \tau)$ is a Hilbert space (see [4]).

First a chain of closed invariant linear subspaces of $T$ is generated mainly by the use of an internal orthogonal projection $P$ of ${ }^{*} H$ onto $H_{\omega}$ where $H_{\omega}$ is an internal, $\omega$-dimensional subspace of ${ }^{*} H, \omega \in{ }^{*} \mathbf{N}-\mathbf{N}$, with the property that any standard point $x \in H$ is infinitesimally close to some point of $H_{\omega}$. Next, it is shown that some member of the chain is nontrivial by using the compactness of $T$ and the properties of *-finite sequences of internal orthogonal projections on ${ }^{*} H$.

Although there is a heavy dependence on the existences of orthogonal projections on $H$, this dependence is not uniform in the two main components of the above procedure. In the first part, i.e., generating the chain of closed invariant linear subspaces for $T$, the three essential properties of projections that were needed are: (1) projections are linear, (2) the norm of the projection of a point $x$ in $H$ is dominated by the norm of $x$, and (3) the projection of a point $x \in H$ is the nearest point, in the subspace, to $x$. To establish, in analogous manner, a chain of closed invariant linear subspaces for a compact operator $T$ when the underlying space $(H, \tau)$ is a topological vector space, we need at least one internal linear transformation $P$ on ${ }^{*} H$ that captures the effects of the three properties stated above. Definition 2.1 gives the conditions for such a transformation and, as we have seen earlier, this definition does give us a means of generating invariant linear subspaces for $T$ (see Proposition 2.10).

Ir the second part, i.e., showing that some member of the chain is nontrivial, only the compactness of $T$ and the nearest point property of projections are used. In other words, it is not necessary to have an internal linear map of ${ }^{*} H$ into $F$ that captures the effects of a nearest point for every internal linear subspace $F$ of ${ }^{*} H$; rather, we need only an internal function that maps ${ }^{*} H$ into $F$ and maintains the essence of a nearest point for each ${ }^{*}$-finite dimensional subspace $F$ of ${ }^{*} H$. Indeed, it is possible to show that such functions exist for a significant class of spaces.

In the following theorem, $\mathscr{F}[H]$ denotes the collection of all functions mapping $H$ into $H$.

THEOREM 3.1. If $(H, \tau)$ is a metrizable topological vector space then there exists a function $\nabla: \mathscr{F} \mathscr{S}(H) \rightarrow \mathscr{F}[H]$ that satisfies the following conditions.

(1) If $F \in \mathscr{F} \mathscr{S}(H)$ then $\nabla(F): H \rightarrow F$.

(2) For each $V \in \mathcal{N}_{\tau}(0)$ and any non zero $x \in H$ there exists a positive $\lambda \in \mathbf{R}$ such that $\nabla(F)(\lambda x) \in V$ for all $F \in \mathscr{F} \mathscr{Y}(H)$. 
(3) If $x \in H$ such that $x \in{ }^{\circ} F$ for $F \in{ }^{*} \mathscr{F} \mathscr{Y}(H)$ then ${ }^{*} \nabla(F)(x)-$ $x \in \mu_{r}(0)$.

\section{(See [6], Theorem II.1.)}

Now the stage is set for the proof of the main result for metrizable topological vector spaces. The plan of attack is straightforward. First a suitable chain of invariant internal linear subspaces for $T^{\prime}=P^{*} T P$ is found. Using Propositions 1.7 and 2.10 we can easily obtain a chain of closed invariant subspaces for the compact operator $T$. Next we must insure that at least one member of the chain is non-trivial which therefore precipitates the result.

THEOREM 3.2. Let $(H, \tau)$ be a complex metrizable topological vector space that satisfies Property 1. If $T \in \mathscr{L}(H)$ is a compact operator then $T$ has at least one nontrivial $\tau$-closed invariant linear subspace of $H$.

Proof. We will assume that $H$ is infinite dimensional and the compact operator $T$ is not null on all of $H$. Also, $P \in * \mathscr{L}(H)$, $H_{\eta} \in * \mathscr{F} \mathscr{Y}(H)$ are determined by Property 1 and $\nabla: \mathscr{F} \mathscr{Y}(H) \rightarrow \mathscr{F}[H]$ is assumed to satisfy the conditions of Theorem 3.1.

Define $T^{\prime}=P^{*} T P$ and let $W \in \mathcal{N}_{\tau}(0)$ such that

$$
T^{\prime}\left[{ }^{*} W\right] \subset \mathrm{ns}_{\tau}\left({ }^{*} H\right)
$$

(see Proposition 2.12). If $T(y) \neq 0$ for $y \in H$ then there exists a positive $\lambda \in \mathbf{R}$ such that

$$
{ }^{*} \nabla(F)(\lambda y) \in{ }^{*} W
$$

for each $F \in * \mathscr{F} \mathscr{S}(H)$ by Condition 2 of Theorem 3.1. Consequently, if we set $x_{0}=\lambda y$ then

$$
T^{\prime}\left(x_{0}\right) \notin \cdot \mu_{\tau}(0)
$$

by Proposition 2.9 and

$$
T^{\prime}\left({ }^{*} \nabla(F)\left(x_{0}\right)\right) \in \mathrm{ns}_{\tau}\left({ }^{*} H\right)
$$

for any $F \in * \mathscr{F} \mathscr{S}(H)$.

Let $T_{\eta}$ denote the restriction of $T^{\prime}$ to $H_{\eta}$. As in [2] and [4], we observe that in a finite-dimensional complex vector space $E$ of dimension $m$, say, any linear operator possesses a finite chain of invariant subspaces 


$$
\{0\}=F_{0} \subset F_{1} \subset \cdots \subset F_{m}=E
$$

where $d\left(F_{j}\right)=j$ for $j=0, \cdots, m$ (recall from $\S 1$ that $d$ is the dimension function defined on $\mathscr{F} \mathscr{S}(H)$ ). Therefore there exists a ${ }^{*}$-finite chain of *-finite dimensional subspaces

$$
\{0\}=F_{0} \subset F_{1} \subset \cdots \subset F_{\omega}=H_{\eta}
$$

such that

$$
\begin{gathered}
T_{\eta}\left[F_{\iota}\right] \subset F_{\iota} \text { for } \iota \in\{0, \cdots, \omega\} \\
* d\left(F_{\iota}\right)=* d\left(F_{\iota-1}\right)+1 \text { for } \iota \in\{1, \cdots, \omega\}
\end{gathered}
$$

where $\omega=* d\left(H_{\eta}\right)$.

Let $Q_{\iota}=* \nabla\left(F_{\iota}\right)$ for $\iota \in\{0, \cdots, \omega\}$ and consider the expression

$$
r_{\iota}=* \rho\left(T^{\prime}\left(x_{0}\right)-T^{\prime} Q_{\iota}\left(x_{0}\right), 0\right), \quad \iota=0,1, \cdots, \omega
$$

where $\rho$ is a translation-invariant metric on $H$ that generates $\tau$. Note that $r_{0}=* \rho\left(T^{\prime}\left(x_{0}\right), 0\right)$ is not an infinitesimal by (3.3) since $F_{0}=$ $\{0\}$. Therefore, $\delta<r_{0}$ for some positive $\delta \in \mathbf{R}$. Since $x_{0} \in H={ }^{\circ} H_{\eta}=$ ${ }^{\circ} F_{\omega}$ implies $x_{0}-Q_{\omega}\left(x_{0}\right) \in \mu_{\tau}(0)$ (see $(2.2),(3,5)$ and Condition 3 of Theorem 3.1) and $T^{\prime}\left[\mu_{\tau}(0)\right] \subset \mu_{\tau}(0)$ implies

$$
T^{\prime}\left(x_{0}\right)-T^{\prime} Q_{\omega}\left(x_{0}\right)=T^{\prime}\left(x_{0}-Q_{\omega}\left(x_{0}\right)\right) \in \mu_{\tau}(0),
$$

it follows that $r_{\omega}$ is an infinitesimal. Consequently, $r_{\omega}<\frac{1}{2} \delta<r_{0}$ which implies

$$
r_{\nu}<\frac{1}{2} \delta \leqq r_{\nu-1}
$$

for some $\nu \in\{1, \cdots, \omega\}$ since $\left\{r_{0}, \cdots, r_{\omega}\right\}$ is *-finite.

Consider ${ }^{\circ} F_{\nu-1}$ and ${ }^{\circ} F_{\nu}$, the $\tau$-standard parts of $F_{\nu-1}$ and $F_{\nu}$ (see (1.3)). Propositions 1.7, 2.10 and expressions (3.5), (3.6) imply ${ }^{\circ} F_{\nu-1}$ and ${ }^{\circ} F_{\nu}$ are $\tau$-closed invariant linear subspaces of $T$ since $T_{\eta}=T^{\prime}$ on $H_{\eta}$. Now ${ }^{\circ} F_{\nu-1}$ cannot coincide with $H$, in particular it cannot include $x_{0}$. For if it did, then $r_{\nu-1}$ would be an infinitesimal (see (3.8) and Condition 3 of Theorem 3.1) contrary to (3.9).

On the other hand, ${ }^{\circ} F_{\nu}$ cannot reduce to $\{0\}$. Indeed, if ${ }^{\circ} F_{\nu}=\{0\}$ then ${ }^{\circ}\left[T^{\prime} Q_{\nu}\left(x_{0}\right)\right]=0$ by (3.4) and (3.6) which would imply $T^{\prime} Q_{\nu}\left(x_{0}\right) \in$ $\mu_{\tau}(0)$. We would then have 


$$
\begin{aligned}
r_{0}-r_{\nu} & ={ }^{*} \rho\left(T^{\prime}\left(x_{0}\right), 0\right)-{ }^{*} \rho\left(T^{\prime}\left(x_{0}\right)-T^{\prime} Q_{\nu}\left(x_{0}\right), 0\right) \\
& \leqq{ }^{*} \rho\left(T^{\prime}\left(x_{0}\right)-\left[T^{\prime}\left(x_{0}\right)-T^{\prime} Q_{\nu}\left(x_{0}\right)\right], 0\right) \\
& ={ }^{*} \rho\left(T^{\prime} Q_{\nu}\left(x_{0}\right), 0\right)<\frac{1}{2} \delta .
\end{aligned}
$$

Therefore, $\delta<r_{0}$ and $r_{0}-r_{\nu}<\frac{1}{2} \delta$ would imply $\frac{1}{2} \delta<r_{\nu}$ contrary to (3.9).

Finally from expressions (3.5), (3.7) and Proposition 1.8, we infer that either ${ }^{\circ} F_{\nu-1}$ or ${ }^{\circ} F_{\nu}$ is nontrivial.

4. Property 2. So far, we still have not shown that compact operators on arbitrary complex topological vector spaces satisfying Property 1 have nontrivial, closed invariant linear subspaces. We will fulfill this obligation in this section. We will do it by the usual mathematical ploy of defining other conditions, collectively called Property 2, that are more convenient. We then establish the result using these conditions and finally we show that spaces satisfying Property 1 also satisfy the new conditions, Property 2 .

In this section, $(H, \tau)$ is an arbitrary complex topological vector space.

Define $\mathcal{M}[\tau]$ as follows: $\rho \in \mathcal{M}[\tau]$ if and only if $\rho$ is a translationinvariant pseudo-metric on $H$ such that for $g_{\rho}: H \rightarrow \mathbf{R}$ defined by $g_{\rho}(x)=\rho(x, 0), x \in H$, we have $g_{\rho}(\lambda x) \leqq g_{\rho}(x)$ for $|\lambda| \leqq 1$ and $g_{\rho}$ is continuous at $0 \in H$. Clearly, $g_{\rho}$ is subadditive and therefore continuous on all of $H$. Furthermore, it can be shown that

$$
\{S(\rho ; \delta) \mid \rho \in \mathcal{M}[\tau], \quad 0<\delta \in \mathbf{R}\}
$$

forms a filter basis for $\mathcal{N}_{\tau}(0)$ where

$$
S(\rho ; \delta)=\{x \in H \mid \rho(x, 0) \leqq \delta\}
$$

(see [9], Theorem 6.7).

For $\rho \in \mathcal{M}[\tau]$ let $\tau_{\rho}$ denote the topology on $H$ generated by $\rho$. Obviously, $\rho \in \mathcal{M}[\tau]$ implies $\tau_{\rho} \subset \tau$ and $\left(H, \tau_{\rho}\right)$ is a complex topological vector space.

DEFINITION 4.1. A topological vector space $(H, \tau)$ is said to satisfy Property 2 if and only if there exists $\mathscr{A} \subset \mathcal{M}[\tau]$ such that the following conditions are fulfilled.

(1) The collection $\{S(\rho ; \delta) \mid \rho \in \mathscr{A}, 0<\delta \in \mathbf{R}\}$ is a filter basis for $\mathcal{N}_{\tau}(\mathbf{0})$.

(2) If $\rho \in \mathscr{A}$ then $\left(H, \tau_{\rho}\right)$ satisfies Property 1. 
THEOREM 4.2. Let $(H, \tau)$ be a complex topological vector space that satisfies Property 2. If $\tau$ is not the chaotic topology and if $T \in \mathscr{L}(H)$ is a compact operator then $T$ has at least one nontrivial, $\tau$-closed invariant linear subspace of $H$.

Proof. Let $T \in \mathscr{L}(H)$ such that $\overline{T[V]}$ is $\tau$-compact for some $V \in \mathcal{N}_{\tau}(0)$. It can be assumed that $V \neq H$ since $\tau \neq\{H, \phi\}$. Let $\mathscr{A} \subset \mathcal{M}[\tau]$ fulfill the conditions of Definition 4.1. Thus, $S\left(\rho ; \delta_{0}\right) \subset V$ for some $\rho \in \mathscr{A}$ and some positive $\delta_{0} \in \mathbf{R}$. Consequently, $\left(H, \tau_{\rho}\right)$ is a complex topological vector space such that $\tau_{\rho} \subset \tau, V \in \mathcal{N}_{\tau_{\rho}}(0)$ and $\left(H, \tau_{\rho}\right)$ satisfies Property 1.

Let $M=\{x \in H \mid \rho(x, 0)=0\}$. If $x \in M$ and $\lambda \in \mathbf{C}$ then

$$
\rho(\lambda x, 0)=\rho\left(\left[\lambda n^{-1}\right] n x, 0\right) \leqq \rho(n x, 0)=0
$$

for $n \in \mathbf{N}$ and $|\lambda|<n$. Consequently, $M$ is a proper $\tau_{\rho}$-closed linear subspace of $H$.

If $\delta \in \mathbf{R}$ is positive then by the $\tau_{\rho}$-continuity of $T$, there exists a positive $\delta_{1} \in \mathbf{R}$ such that

$$
T[M] \subset T\left[S\left(\rho ; \delta_{1}\right)\right] \subset S(\rho ; \delta)
$$

therefore, $T[M] \subset M$. So if $M \neq\{0\}$ then $M$ is a nontrivial, $\tau$-closed invariant linear subspace of $T$ since $\tau_{\rho}$ is weaker than $\tau$.

If $M=\{0\}$ then $\left(H, \tau_{\rho}\right)$ is a complex metrizable topological vector space that satisfies Property 1. By Theorem 3.2 there exists a nontrivial $\tau_{\rho}$-closed, and thus $\tau$-closed, linear subspace $F \subset H$ such that $T[F] \subset F$.

Next, we show that Property 1 implies Property 2. The basic idea is to start with an arbitrary balanced neighborhood of zero and use Condition 2 of Definition 2.1 to obtain a sequence of balanced neighborhoods of zero that generates a pseudo-metric linear topology satisfying Property 1.

TheOREm 4.3. If a topological vector space $(H, \tau)$ satisfies Property 1 then $(H, \tau)$ satisfies Property 2.

Proof. Let $P \in * \mathscr{L}(H), H_{\eta} \in * \mathscr{F} \mathscr{P}(H)$ be determined by Property 1 and let $V \in \mathcal{N}_{\tau}(0)$ be balanced. By Condition 2 of Definition 2.1 there exists $W \in \mathcal{N}_{\tau}(0)$ such that $P\left[{ }^{*} W\right] \subset^{*} V$. Also, there exists a balanced $V_{2} \in \mathcal{N}_{\tau}(0)$ such that

$$
V_{2}+V_{2}+V_{2} \subset V \cap W
$$


which implies $P\left[{ }^{*} V_{2}\right] \subset^{*} V$. Therefore define

$$
\left\{V_{n}\right\}_{n=1}^{\infty} \subset \mathcal{N}_{\tau}(0)
$$

inductively as follows: $V_{1}=V$, for $n \in \mathbf{N}_{+}$let $V_{n+1} \in \mathcal{N}_{\tau}(0)$ be a balanced neighborhood such that

$$
V_{n+1}+V_{n+1}+V_{n+1} \subset V_{n}
$$

and

$$
P\left[* V_{n+1}\right] \subset^{*} V_{n}
$$

Consequently, there exists an unique topology $\tau_{V}$ on $H$ such that $\left(H, \tau_{V}\right)$ is a topological vector space and $\left\{V_{n}\right\}_{n=1}^{\infty}$ is a filter basis for $\mathcal{N}_{\tau_{v}}(0)$ (see [8], Theorem 2.3.1).

Conditions 1 and 2 of Definition 2.1 are satisfied by the definitions of $P, H_{\eta}$ and $\left\{V_{n}\right\}_{n=1}^{\infty}$. Also $\tau_{V}$ is weaker than $\tau$ by (4.4) which implies $\mu_{\tau}(0) \subset \mu_{\tau_{v}}(0)$ ([6], Chapter I, §2). Thus, $P(x)-x \in \mu_{\tau}(0)$ implies $P(x)-x \in \mu_{\tau_{v}}(0)$ for $x \in H$. Therefore, $\left(H, \tau_{V}\right)$ satisfies Property 1 .

Using (4.4) and (4.5) it can be shown that some $\rho \in \mathcal{M}[\tau]$ generates $\tau_{V}$ (see [9], Theorem 6.7 or [8], Theorem 2.6.1). Therefore, if we define

$$
\mathscr{A}=\left\{\rho \in \mathcal{M}[\tau] \mid \rho \text { generates } \tau_{V} \text { for balanced } V \in \mathcal{N}_{\tau}(0)\right\}
$$

then $\mathscr{A}$ fulfills the conditions of Definition 4.1 ; that is, $(H, \tau)$ satisfies Property 2.

THEOREM 4.7. Let $(H, \tau)$ be a complex topological vector space that satisfies Property 1 . If $\tau$ is not the chaotic topology and if $T \in \mathscr{L}(H)$ is a compact operator then $T$ has at least one nontrivial $\tau$-closed invariant linear subspace of $H$.

Proof. Theorems 4.3 and 4.2 .

5. Metrizable topological vector spaces with Schauder basis. In this section we examine the conditions needed for a metrizable topological vector space with a Schauder basis to satisfy Property 1. In particular we will show that a Fréchet space (i.e., a complete, metrizable topological vector space) with a Schauder basis satisfies Property 1.

In this section, $(H, \tau)$ is assumed to be a metrizable complex topological vector space. 
If $\left\{x_{j}\right\}$ is a sequence in $H$ then $\mathscr{F}_{r}\left[x_{j} ; H\right]$ will denote the filter, on $H$, generated by $\left\{\left\{x_{j}\right\}_{j=n}^{x} \mid n \in \mathbf{N}_{+}\right\}$.

Definition 5.1. Let $(H, \tau)$ be a metrizable complex topological vector spaces. A sequence $\left\{e_{i}\right\} \subset H$ of distinct nonzero elements is a Schauder basis for $(H, \tau)$ if and only if for each $x \in H$ there exists an unique sequence $\left\{\xi_{i}\right\} \subset \mathbf{C}$ such that $\mathscr{F}_{r}\left[\sum_{i=1}^{j} \xi_{1} e_{i} ; H\right]$ converges to $x$ with respect to $\tau$.

Assume that a sequence $\left\{e_{i}\right\} \subset H$ is a Schauder basis for $(H, \tau)$. Let

$$
E=\operatorname{sp}\left(\left\{e_{l}\right\}\right) \text { and } E_{j}=\operatorname{sp}\left(e_{1}, \cdots, e_{j}\right)
$$

for $j \in \mathbf{N}_{+}$, i.e., $E$ and $E_{j}$ are the smallest linear subspaces of $H$ that contain $\left\{e_{i}\right\}$ and $\left\{e_{1}, \cdots, e_{j}\right\}$ respectively. Clearly, $E=\cup_{j=1}^{\infty} E_{j}$ and $\left\{E_{j} \mid j \in \mathbf{N}_{+}\right\} \subset \mathscr{F} \mathscr{S}(H)$. Furthermore it is easily seen that $H=\bar{E}=$ $\cup_{j=1}^{\infty} E_{j}$ from which we conclude $(H, \tau)$ is separable. We will call $\left\{E_{j} \mid j \in \mathbf{N}_{+}\right\}$the sequence of coordinate spaces generated by $\left\{e_{l}\right\}$.

If, for each $i \in \mathbf{N}_{+}$, we define $\pi_{i}: H \rightarrow \mathbf{C}$ by $\pi_{i}(x)=\xi_{i}$ for $x \in H$ and $\left\{\xi_{i}\right\}$, the unique complex sequence determined for $x$ by $\left\{e_{i}\right\}$, then it can be shown that $\pi_{i}$ is a linear functional on $H$ that maps $e_{i}$ to 1 and $e_{j}$ to 0 for $j \in \mathbf{N}_{+}$and $j \neq i$.

For each $j \in \mathbf{N}_{+}$define $P_{l}: H \rightarrow E_{l}$ by

$$
P_{j}(x)=\sum_{i=1}^{j} \pi_{i}(x) e_{i}
$$

for $x \in H$. Consequently, $P_{j} \in \mathscr{L}(H)$, i.e., $P_{\mathrm{J}}$ is a linear transformation and $P_{j}(y)=y$ for $y \in E_{l}$. We will call $\left\{P_{j} \mid j \in \mathbf{N}_{+}\right\}$the sequence of projections generated by $\left\{e_{i}\right\}$.

Proposition 5.4. Let $\left\{e_{i}\right\}$ be a Schauder basis for $(H, \tau)$ and let $\left\{P_{1} \mid j \in \mathbf{N}_{+}\right\}$be the sequence of projections generated by $\left\{e_{i}\right\}$. If there exists a filter basis $\mathscr{B} \subset \mathcal{N}_{\tau}(0)$ such that $P_{j}[V] \subset V$ for $V \in \mathscr{B}$ and $P_{j} \in\left\{P_{j} \mid j \in \mathbf{N}_{+}\right\}$then $(H, \tau)$ satisfies Property 1.

(See [6], Proposition III.1.)

Let $\rho$ be a translation-invariant metric on $H$ that generates $\tau$. We will call $\left\{e_{1}\right\}$ a monotone Schauder basis (with respect to $\rho$ ) if and only if the sequence $\left\{\rho\left(P_{j}(x), 0\right) \mid j \in \mathbf{N}_{+}\right\}$is monotonely increasing for each $x \in H$, that is, if $i, j \in \mathbf{N}_{+}$and $i \leqq j$ then $\rho\left(P_{i}(x), 0\right) \leqq \rho\left(P_{j}(x), 0\right)$ for each $x \in H$. 
Proposition 5.5. If $(H, \tau)$ has a monotone Schauder basis then $(H, \tau)$ satisfies Property 1.

Proof. Let $\rho$ be a translation-invariant metric on $H$ that generates $\tau$ and let $\left\{e_{l}\right\}$ be a monotone Schauder basis for $(H, \tau)$ with respect to $\rho$. If $x \in H$ then $\rho\left(P_{f}(x), 0\right) \leqq \rho(x, 0)$ for each $j \in \mathbf{N}_{+}$by the continuity of $\rho$. Therefore, if $\delta \in \mathbf{R}$ is positive then $x \in S(\rho ; \delta)$ implies $\rho\left(P_{j}(x), 0\right) \leqq \rho(x, 0) \leqq \delta \quad$ for $j \in \mathbf{N}_{+}$. Consequently $P_{j}[S(\rho ; \delta)] \subset$ $S(\rho ; \delta)$ for each $P_{j} \in\left\{P_{j} \mid j \in \mathbf{N}_{+}\right\}$. We conclude that $(H, \tau)$ satisfies Property 1 from Proposition 5.4.

In the following theorem, a Fréchet space is a complete metrizable topological vector space.

Theorem 5.6. Let $(H, \tau)$ be a Fréchet space. If $(H, \tau)$ has a Schauder basis then $(H, \tau)$ satisfies Property 1.

Proof. Let $\left\{e_{l}\right\}$ be a Schauder basis for $(H, \tau)$ and let $\left\{P_{j} \mid j \in \mathbf{N}_{+}\right\}$ be the sequence of projections generated by $\left\{e_{i}\right\}$. Also, let $\rho$ be a translation-invariant metric on $\boldsymbol{H}$ that generates $\tau$. Define $\boldsymbol{H} \rightarrow \mathbf{R}$ as follows:

$$
\rho_{0}(x, y)=\sup \left\{\rho\left(P_{j}(x-y), 0\right) \mid j \in \mathbf{N}_{+}\right\}
$$

for $x, y \in H$. Since $(H, \tau)$ is complete, it can be shown that $\rho_{0}$ is a translation-invariant metric on $H$ that generates $\tau$ (see [13], Theorem 11.4.1). Furthermore, $\left\{e_{1}\right\}$ is a monotone Schauder basis with respect to $\rho_{0}$. Therefore, $(H, \tau)$ satisfies Property 1 by Proposition 5.5.

Corollary 5.7. If $(H, \tau)$ is a complex Fréchet space with a Schauder basis and if $T \in \mathscr{L}(H)$ is a compact operator then $T$ has at least one nontrivial closed invariant linear subspace of $H$.

Proof. Theorems 5.6 and 3.2.

EXAmple 5.8. Let $\mathscr{S} q(\mathbf{C})$ be the collection of all complex sequences and let $\left(\xi_{j}\right)$ symbolize an element of $\mathscr{S} q(\mathbf{C})$, i.e., $\xi_{j} \in \mathbf{C}$ for $j \in \mathbf{N}_{+}$. The set $\mathscr{S} q(\mathbf{C})$ is a complex vector space under the usual pointwise definition of addition and scalar multiplication.

Let $\left\{p_{j}\right\}$ be a sequence of real numbers satisfying the inequalities $0<p_{j} \leqq 1$ for each $j \in \mathbf{N}_{+}$and define

$$
\ell\left(p_{j}\right)=\left\{\left.\left(\xi_{J}\right) \in \mathscr{S} q(\mathbf{C})\left|\sum_{j=1}^{\infty}\right| \xi_{j}\right|^{p_{i}}<\infty\right\}
$$


Since the identities $|\lambda+\beta|^{p} \leqq|\lambda|^{p}+|\beta|^{p}$ and $|\lambda \beta|^{p} \leqq \max$ $(1,|\lambda|)|\beta|^{p}$ are valid for all $\lambda, \beta \in \mathbf{C}$ and $0<p \leqq 1$, it follows that $\ell\left(p_{j}\right)$ is a vector subspace of $\mathscr{S} q(\mathbf{C})$. Also, we can define a translationinvariant metric $\rho\left(p_{j}\right)$ on $\ell\left(p_{j}\right)$ as follows:

$$
\rho\left(p_{j}\right)(x, y)=\sum_{j=1}^{\infty}\left|\xi_{j}-\zeta_{j}\right|^{p_{j}}
$$

for $x=\left(\xi_{j}\right), y=\left(\zeta_{j}\right) \in \ell\left(p_{j}\right)$. We will let $\tau\left(p_{j}\right)$ denote the topology on $\ell\left(p_{j}\right)$ generated by $\rho\left(p_{j}\right)$.

S. Simons made an extensive study of $\left(\ell\left(p_{j}\right), \tau\left(p_{j}\right)\right)$ in [12]. Among the facts he established are the following.

(5.9) $\quad\left(\ell\left(p_{j}\right), \tau\left(p_{j}\right)\right)$ is a Fréchet space.

(5.10) $\quad\left(\ell\left(p_{j}\right), \tau\left(p_{j}\right)\right)$ is locally convex if and only if $\ell\left(p_{j}\right)=\ell^{\prime}$.

(5.11) $\quad\left(\ell\left(p_{j}\right), \tau\left(p_{j}\right)\right)$ is locally bounded if and only if $\liminf p_{j}>0$.

(See [12], Lemma 1, Theorems 5 and 6.)

If, for $j \in \mathbf{N}_{+}$, we define $e_{j}=\left(\xi_{k}\right)$ such that $\xi_{j}=1$ and $\xi_{k}=0$ for $k \in \mathbf{N}_{+}$and $k \neq j$ then $\left\{e_{j}\right\}$ is a Schauder basis for $\left(\ell\left(p_{j}\right), \tau\left(p_{j}\right)\right)$ by definition of $\rho\left(p_{j}\right)$. Therefore, $\left(\ell\left(p_{j}\right), \tau\left(p_{j}\right)\right)$ satisfies Property 1 by (5.9) and Theorem 5.6. Furthermore, if $p_{j} \rightarrow 0$ as $j \rightarrow \infty$ then $\ell\left(p_{j}\right) \neq \ell^{1}$ since $x_{0}=\left(j^{-q_{i}}\right) \notin \ell\left(p_{j}\right)$, where $q_{j}=p_{j}^{-1}$, and $x_{0} \in \ell^{1}$. Consequently, from (5.10) and (5.11) we can infer that if $\left\{p_{j}\right\}$ converges to 0 then $\left(\ell\left(p_{j}\right), \tau\left(p_{j}\right)\right)$ is neither locally convex nor locally bounded. In particular, the space $\left(\ell\left(j^{-1}\right), \tau\left(j^{-1}\right)\right)$ is not locally convex and not locally bounded.

EXAMPLE 5.12. Let $A=\left[a_{i j}\right]$ and $B=\left[b_{i j}\right]$ be two infinite matrices of real numbers such that $0<a_{i j}$ and $0<b_{i j} \leqq 1$ for $i, j \in \mathbf{N}_{+}$. Define $\ell(A, B)$ in the following manner. If $x=\left(\xi_{j}\right)$ is a sequence of complex numbers then $x \in \ell(A, B)$ if and only if

$$
\sum_{j=1}^{\infty} a_{i j}\left|\xi_{j}\right|^{b_{i j}}<\infty
$$

for each $i \in \mathbf{N}_{+}$. Using the same arguments of Example 5.8, it can be shown that $\ell(A, B)$ is a complex vector space.

If we define 


$$
W_{n}=\left\{\left.\left(\xi_{j}\right) \in \ell(A, B)\left|\sum_{j=1}^{\infty} a_{i j}\right| \xi_{j}\right|^{b_{i j}} \leqq n^{-1} \quad \text { for } \quad i=1, \cdots, n\right\}
$$

for each positive integer $n$, then there exists an unique topology $\tau(A, B)$ on $\ell(A, B)$ such that $(\ell(A, B), \tau(A, B))$ is a topological vector space and $\left\{W_{n}\right\}_{n=1}^{\infty}$ is a filter basis for the $\tau(A, B)$-neighborhoods of zero. Indeed, it can be shown that $(\ell(A, B), \tau(A, B))$ is a Fréchet space having $\left\{e_{j}\right\}$ (see Example 5.8) as a Schauder basis ([6], Propositions IV.2 and IV.3). Consequently, $(\ell(A, B), \tau(A, B))$ satisfies Property 1 by Theorem 5.6.

Note that the spaces $\left(\ell\left(p_{j}\right), \tau\left(p_{j}\right)\right)$ of Example 5.8 form a subcollection of the spaces $(\ell(A, B), \tau(A, B))$, i.e., let $a_{i j}=1$ for all $i, j \in \mathbf{N}_{+}$and let $b_{i j}=p_{j}$ for each $i \in \mathbf{N}_{+}$. However, it is possible to define a subclass of the spaces $(\ell(A, B), \tau(A, B))$ that is neither locally convex nor locally bounded and different from the collection $\left(\ell\left(p_{1}\right), \tau\left(p_{j}\right)\right)$.

We will say that $\left[A: B:\left\{q_{i}\right\}\right]$ satisfies p.3, where $A=\left[a_{i j}\right]$ and $B=\left[b_{i j}\right]$ are infinite matrices of real numbers and $\left\{q_{i}\right\}$ is a sequence of real numbers, if and only if

$$
\begin{gathered}
a_{i j}=1 \text { and } 0<q_{i+1}<q_{i}<1 \text { for all } i, j \in \mathbf{N}_{+}, \\
q_{2 i}<b_{i, j+1} \leqq b_{i j}<q_{2 i-1} \text { for all } i, j \in \mathbf{N}_{+} .
\end{gathered}
$$

Now if $\left[A: B:\left\{q_{i}\right\}\right]$ satisfies p.3, then $(\ell(A, B), \tau(A, B))$ is not locally convex, not locally bounded and is distinct from $\left(\ell\left(p_{j}\right), \tau\left(p_{j}\right)\right)$ for any sequence $\left\{p_{i}\right\} \subset(0,1]$ ([6], Propositions IV.4, IV.5 and IV.6).

In particular, if we let $\left(\ell\left[i^{-1}\right], \tau\left[i^{-1}\right]\right)$ symbolize the $(\ell(A, B)$, $\tau(A, B)$ ) space where $a_{i j}=1$ and $b_{i j}=i^{-1}$ for $i, j \in N_{+}$then $\ell\left(i^{-1}\right)$, of Example 5.8, is a proper subset of $\ell\left[i^{-1}\right]$ and $\tau\left[i^{-1}\right]$ induces a weaker topology on $\ell\left(i^{-1}\right)$ than $\tau\left(i^{-1}\right)$.

\section{REFERENCES}

1. N. Aronszajn and K. Smith, Invariant subspaces of completely continuous operators, Ann. of Math., 60 (1954), 345-350.

2. A. R. Bernstein, Invariant subspaces of polynomially compact operators on Banach space, Pacific J. of Math., 21 (1967), 445-464.

3. A. R. Bernstein, Invariant subspaces for certain commuting operators on Hilbert space, Ann. of Math., 95 (1972), 253-260.

4. A. R. Bernstein and A. Robinson, Solution of an invariant subspace problem of K. T. Smith and P. R. Halmos, Pacific J. of Math., 16 (1966), 421-431.

5. N. Dunford and J. T. Schwartz, Linear Operators, Part II, Interscience Publishers, New York, 1963.

6. A. D. Grainger, On nonstandard topological vector spaces and invariant subspaces of compact operators, Doctoral Dissertation, University of Maryland, College Park, Maryland, 1973. 
7. C. W. Henson and L. C. Moore Jr., The nonstandard theory of topological vector spaces, Trans. Amer. Math. Soc., 172 (1972), 405-435.

8. J. Horváth, Topological Vector Spaces and Distributions Volume I, Addison Wesley, Reading, Mass., 1966.

9. J. L. Kelly and I. Namiaka, Linear Topological Spaces, Van Nostrand, Princeton, New Jersey, 1963.

10. W. A. J. Luxemburg, A general theory of monads, in W. A. J. Luxemburg, ed., Applications of Model Theory to Algebra, Analysis and Probability, Holt, Rinehart and Winston, New York (1969), 18-86.

11. A. Robinson, Non-standard Analysis, North-Holland, Amsterdam, 1966.

12. S. Simons, The sequence spaces $\ell\left(p_{\nu}\right)$ and $M\left(p_{\nu}\right)$, Proc. London Math. Soc., (3) 15 (1965), $422-436$.

13. A. Wilansky, Functional Analysis, Blaisdell, New York, 1964.

Received November 16, 1973 and in revised form June 12, 1974. These results were obtained in the author's 1973 doctoral dissertation written at the University of Maryland under the direction of Allen R. Bernstein. 



\section{PACIFIC JOURNAL OF MATHEMATICS \\ EDITORS}

\author{
Richard ARENS (Managing Editor) \\ University of California \\ Los Angeles, California 90024
}

\author{
R. A. Beaumont \\ University of Washington \\ Seattle, Washington 98105
}

\section{J. DugundJI}

Department of Mathematics

University of Southern California

Los Angeles, California 90007

D. Gilbarg and J. Milgram

Stanford University

Stanford, California 94305

\section{ASSOCIATE EDITORS}
E. F. BECKENBACH
B. H. NeumanN
F. WOLF
K. YoshidA

\section{SUPPORTING INSTITUTIONS}

\author{
UNIVERSITY OF BRITISH COLUMBIA \\ CALIFORNIA INSTITUTE OF TECHNOLOGY \\ UNIVERSITY OF CALIFORNIA \\ MONTANA STATE UNIVERSITY \\ UNIVERSITY OF NEVADA \\ NEW MEXICO STATE UNIVERSITY \\ OREGON STATE UNIVERSITY \\ UNIVERSITY OF OREGON \\ OSAKA UNIVERSITY
}

\author{
UNIVERSITY OF SOUTHERN CALIFORNIA \\ STANFORD UNIVERSITY \\ UNIVERSITY OF TOKYO \\ UNIVERSITY OF UTAH \\ WASHINGTON STATE UNIVERSITY \\ UNIVERSITY OF WASHINGTON \\ AMERICAN MATHEMATICAL SOCIETY
}

The Supporting Institutions listed above contribute to the cost of publication of this Journal, but they are not owners or publishers and have no responsibility for its contents or policies.

Mathematical papers intended for publication in the Pacific Journal of Mathematics should be in typed form or offset-reproduced (not dittoed), double spaced with large margins. Underline Greek letters in red, German in green, and script in blue. The first paragraph or two must be capable of being used separately as a synopsis of the entire paper. Items of the bibliography should not be cited there unless absolutely necessary, in which case they must be identified by author and Journal, rather than by item number. Manuscripts, in duplicate, may be sent to any one of the four editors. Please classify according to the scheme of Math. Reviews, Index to Vol. 39. All other communications should be addressed to the managing editor, or Elaine Barth, University of California, Los Angeles, California, 90024.

100 reprints are provided free for each article, only if page charges have been substantially paid. Additional copies may be obtained at cost in multiples of 50 .

The Pacific Journal of Mathematics is issued monthly as of January 1966. Regular subscription rate: $\$ 72.00$ a year (6 Vols., 12 issues). Special rate: $\$ 36.00$ a year to individual members of supporting institutions.

Subscriptions, orders for back numbers, and changes of address should be sent to Pacific Journal of Mathematics, 103 Highland Boulevard, Berkeley, California, 94708.

PUBLISHED BY PACIFIC JOURNAL OF MATHEMATICS, A NON-PROFIT CORPORATION Printed at Jerusalem Academic Press, POB 2390, Jerusalem, Israel.

$$
\begin{gathered}
\text { Copyright (c) } 1975 \text { Pacific Journal of Mathematics } \\
\text { All Rights Reserved }
\end{gathered}
$$




\section{Pacific Journal of Mathematics}

Vol. 56, No. 2 December, 1975

Ralph Alexander, Generalized sums of distances .................. 297

Zvi Arad and George Isaac Glauberman, A characteristic subgroup of a group of odd order ............................... 305

B. Aupetit, Continuité du spectre dans les algèbres de Banach avec involution ........................................ 321

Roger W. Barnard and John Lawson Lewis, Coefficient bounds for some classes of starlike functions . ...........................

Roger W. Barnard and John Lawson Lewis, Subordination theorems for some classes of starlike fumctions . .................... 333

Ladislav Bican, Preradicals and injectivity .................. 367

James Donnell Buckholtz and Ken Shaw, Series expansions of analytic

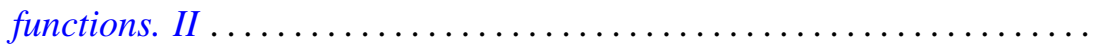

Richard D. Carmichael and E. O. Milton, Distributional boundary values in

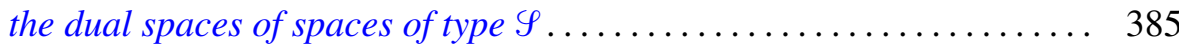

Edwin Duda, Weak-unicoherence ............................ 423

Albert Edrei, The Padé table of functions having a finite number of essential singularities ........................................

Joel N. Franklin and Solomon Wolf Golomb, A function-theoretic approach to the study of nonlinear recurring sequences ................ 455

George Isaac Glauberman, On Burnside's other $p^{a} q^{b}$ theorem 469

Arthur D. Grainger, Invariant subspaces of compact operators on topological vector spaces .........................

Jon Craig Helton, Mutual existence of sum and product integrals .

Franklin Takashi Iha, On boundary functionals and operators with finite-dimensional null spaces

Gerald J. Janusz, Generators for the Schur group of local and global number fields

A. Katsaras and Dar-Biau Liu, Integral representations of weakly compact operators.

W. J. Kim, On the first and the second conjugate points .

Charles Philip Lanski, Regularity and quotients in rings with involution ....

Ewing L. Lusk, An obstruction to extending isotopies of piecewise linear manifolds.

Saburou Saitoh, On some completenesses of the Bergman kernel and the Rudin kernel..

Stephen Jeffrey Willson, The converse to the Smith theorem for $Z_{p}$-homology spheres. 